which gave only transient discharges to maintained pressure, while those with larger fields fired repetitively. On the face the areas of skin represented most frequently were situated around the lips and on the nose; some of the larger receptive fields appeared to cross the midline. In the hand, the tips of the fingers and thumb were commonly involved. The latencies of 22 'hand' units, following electrical stimulation of the contralateral median nerve at the wrist, ranged from 13 to $19 \mathrm{msec}$. Preliminary single-unit studies had failed to demonstrate any gross changes in evoked potentials after various destructive procedures.

\section{TUBERCULOUS OSTEITIS OF THE SKULL}

JOHN MILES and BRODIE HUGHES (Birmingham) stated that tuberculosis affecting the skull had always been a relatively unusual occurrence, comprising only $1 \%$ of all tuberculous osteitis. Little had been seen of it in the Western hemisphere for perhaps 30 years. With the recent expansion of immigration to this country from areas where tuberculosis was still endemic, cranial osteitis, together with the more common forms of tuberculosis, was likely to be encountered more often.

Four cases of tuberculosis of the skull were described two of which were interesting because of neurological symptoms and signs, reputedly uncommon even when this condition was less rare. All four patients were immigrants; three presented with head pain and fluctuant swelling of the scalp. Radiographs revealed destruction of the underlying bone and they were treated by local craniectomy with appropriate antibiotics. A 15-year-old Pakistani boy developed intractable ulceration of the mouth and throat, became blind in the right eye, and lost the nasal field of his left eye. In addition he had extensive ophthalmoplegias and bulbar paresis affecting the palate, tongue, and vocal cords. Radiographs showed complete destruction of the basisphenoid with widening of the retropharyngeal space, and aspiration of the retropharyngeal abscess revealed the tuberculous nature of the infection.

Surgery for the removal of necrotic bone was still considered advisable and certainly in the treatment of massive extradural collections of pus. It was thought that chemotherapy alone would probably suffice in many cases and there was little alternative when the base of the skull was primarily involved.

Study of the literature had revealed that tuberculous osteitis was evidently a relatively benign condition, as 60 of the 87 cases operated upon up to 1933 - that is, before effective chemotherapy-were said to be completely cured by surgery.

\section{CEREBELLAR HAEMANGIOBLASTOMAS}

JOHN GLEAVE (Cambridge) reviewed those cases of haemangioblastoma which had been treated at Cambridge since 1963. He stressed the importance of vertebral angiography in diagnosis and in planning treatment and commented on certain problems of anaesthesia for posterior fossa surgery.

\author{
HYPERBARIC OXYGENATION: A TREATMENT IN \\ NEUROSURGERY
}

K.-H. HOLBACH, F.-K. SCHRÖDER, G. DATENÉ, and H. DOHR (Bonn) reported their experiences in the use of hyperbaric oxygen (HO) in the treatment of traumatic post-operative and cerebrovascular cases. Their object was to institute HO therapy during the acute phase of concomitant brain oedema in an attempt to diminish secondary hypoxic damage. Patients were exposed to oxygen pressures between 2 and 3 atmospheres for periods from 30 minutes to one hour. Blood gases, $\mathrm{pH}$, lactate and pyruvate concentrations were measured; ECG and EEG recordings were made and in some cases blood pressure, pCSF, and rCBF of the affected brain tissue. Fifty-two patients were studied; 23 who had suffered severe head injuries were submitted to HO therapy on 40 occasions. On $50 \%$ of these occasions some improvement clinically was noticed -in about half of these, a marked improvement. On the second day after treatment 11 patients continued to improve, five were unchanged, and seven deteriorated. Treatment of 21 patients suffering from cerebrovascular disease produced very similar results. The remaining groups of cases were too small for analysis, but in the total assessment 22 of 52 cases showed continuing improvement, while 30 remained unchanged or deteriorated. In the cerebrovascular disease group apparent clinical improvement during $\mathrm{HO}$ therapy was comparatively rare, although a greater proportion showed improvement in the EEG record.

Although the ultimate outcome in the $42 \%$ of these patients who showed improvement with $\mathrm{HO}$ was strikingly better than in those who did not, the authors attributed this to a considerable extent to the great variability of cerebral damage and complications. They also called attention to the often transitory nature of the improvement following HO therapy and stressed the importance of a continuous EEG recording to monitor treatment.

The results of their biochemical investigations indicated that during $\mathrm{HO}$ therapy there was a rise of $\mathrm{aPO}_{2}$, while $\mathrm{PCO}_{2}$ and $\mathrm{pH}$ remained within normal limits. There was also a decrease in lactate and pyruvate concentrations indicating a reduction of anaerobic in favour of aerobic metabolism with greater energy production. Cerebral blood flow studies suggested that perfusion was influenced by varying degrees of cerebral vasoconstriction under hyperbaric $\mathrm{O}_{2}$. It was suggested that, in addition to a significant elevation of $\mathrm{aPO}_{2}$, cerebral vasoconstriction might at times be a favourable factor, as when brain swelling after injury had been associated with vasodilatation. In other circumstances, it was admitted that cerebral vasoconstriction might be a harmful influence. The authors concluded that HO therapy had been shown to be useful in certain cases and was a method deserving further study.

\section{OPERATIVE ANGIOGRAPHY}

J. W. TURNER and K. W. GROSSART (Glasgow) have employed angiography in selected cases during the course of cerebrovascular surgery. A radiolucent headrest was 